Horizons philosophiques

\title{
Clément Rosset ou La joie tragique (entretien)
}

\section{Sébastien Charles}

Volume 9, numéro 2, printemps 1999

La philosophie à portée de voix

URI : https://id.erudit.org/iderudit/801134ar

DOI : https://doi.org/10.7202/801134ar

Aller au sommaire du numéro

\section{Éditeur(s)}

Collège Édouard-Montpetit

\section{ISSN}

1181-9227 (imprimé)

1920-2954 (numérique)

Découvrir la revue

\section{Citer ce document}

Charles, S. (1999). Clément Rosset ou La joie tragique (entretien). Horizons philosophiques, 9(2), 91-108. https://doi.org/10.7202/801134ar d'utilisation que vous pouvez consulter en ligne.

https://apropos.erudit.org/fr/usagers/politique-dutilisation/ 


\title{
CLÉMENT ROSSET OU LA JOIE TRAGIQUE1
}

\author{
"La philosophie fera en sorte que celui qui aura obéi à \\ ses lois aura toujours des armes contre la fortune, qu'il \\ trouvera en lui-même tous les secours nécessaires à \\ la vie heureuse, de manière à être toujours heureuX". \\ CICÉRON
}

De nos jours Derrida, Deleuze et Foucault rencontrent encore un immense succès outre-Atlantique et sont à la source de nombreuses écoles de pensée dans lesquelles affluent un grand nombre de disciples. Votre pensée, qui s'est élaborée à la même époque, n'a pas, elle, donné lieu à un phénomène de ce genre. Comment expliquez-vous le fait que vous soyez lu sans être adoré, compris sans être adulé ? Est-ce là un état de fait conforme à votre "pensée terroriste" qui se veut lucide et sans compromission aucune avec les courants philosophiques à la mode ?

Je crois que vous posez la question de manière inexacte, ou du moins les faits que vous invoquez ne sont pas tout à fait exacts. II est évident que j'ai été beaucoup moins lu - ou du moins beaucoup moins acheté parce que je ne sais pas combien de gens ont vraiment lu en-deçà ou au-delà de l'Atlantique un certain nombre de livres de ces philosophes, et notamment ceux qui étaient particulièrement gros ou indigestes - et que je n'ai pas obtenu le même succès au niveau des ventes et de la notoriété (articles dans les journaux, émissions diverses et

1. Cet entretien paraît sous une forme quelque peu différente dans Une fin de siècle philosophique. Entretiens avec André Comte-Sponville, Marcel Conche, Luc Ferry, Gilles Lipovetsky, Michel Onfray et Clément Rosset, Montréal, Liber, 1999. II n'aurait pu avoir lieu sans l'appui des membres du Fonds de Développement Académique de l'Université d'Ottawa que nous tenons à remercier chaleureusement ici. 
autres choses dans ce genre-là). En revanche, si mon public a été limité, il a toujours été très enthousiaste. Si j'ai moins de lecteurs, ils sont répartis dans le monde entier (ce qui m'étonne toujours un peu) et sont très attachés à ce que j'écris et mettent mes ouvrages très au-dessus des grands noms auxquels vous avez fait allusion. Ce n'est donc pas du même genre de notoriété qu'il s'agit parce que notre public diffère et que nous ne cheminons pas sur les mêmes pistes ni ne combattons dans les mêmes arènes et avec les mêmes juges. C'est pourquoi cette question se résout d'elle-même par la divergence de nos écritures, de nos objectifs et de nos lecteurs. Je parle ici bien sûr de ceux de mes lecteurs qui ont pris beaucoup de plaisir à me lire, mais c'est le cas de la grande majorité de ceux qui me lisent, même si, évidemment, il y a des gens qui détestent avec beaucoup de violence intellectuelle ce que je fais, pour la raison du contenu essentiellement.

Ce que je voulais aussi mentionner c'est le fait que votre philosophie n'ait pas été récupérée par l'intelligentsia nordaméricaine. N'est-ce pas parce qu'elle ne s'y prêtait guère ?

Ma pensée a intéressé des philosophies très variées mais il est certain qu'elle a été plus lue et plus traduite dans des pays méridionaux, latins ou orientaux (Chine et Japon par exemple), plus rarement en Allemagne et exceptionnellement du côté anglo-saxon'2, fait qui mérite à lui seul d'être souligné. Cela me semble s'expliquer aisément par le fait que les tendances philosophiques anglo-saxonnes (la philosophie analytique en particulier) vont résolument dans un sens qui diffère radicalement de ce que j'écris, ce qui fait que les Anglo-Saxons n'ont rien à attendre de mon œuvre comme je n'ai rien à attendre de ce qu'ils font eux-mêmes. Une des preuves de ceci est le fait que le succès aux États-Unis de certains des grands noms que vous évoquiez au début de l'entretien, et on pourrait en citer d'autres, a trouvé un écho particulier dans des milieux littéraires

2. Le seul ouvrage de Clément Rosset disponible en anglais est Joyful Cruelty. Towards a Philosophy of the Real. Toutes nos références aux ouvrages de Clément Rosset sont données intégralement en annexe. 
(les départements de lettres des Universités) plutôt que philosophiques.

Je regrette un peu néanmoins de n'avoir pas percé en Angleterre parce que je trouve qu'il existe un certain nombre de points communs entre ma pensée et la philosophie anglaise, non l'analytique, vous vous en doutez, mais celle des XVIIle et $\mathrm{XIXe}$ siècles qui a été la première à lire Montaigne et à en faire quasiment un philosophe anglais, de même qu'elle a considéré Haendel comme un compositeur anglais. Mon propre amour de Montaigne m'aurait fait attendre un peu plus d'écho en Angleterre, mais cet écho aura peut-être lieu un jour car, selon l'expression d'Althusser, «l'avenir dure longtemps».

Dans L'anti-nature, vous avancez l'idée selon laquelle toute philosophie artificialiste "n'a pu se produire qu'à la faveur des carences de la philosophie proprement dite»3. Cela est-il vrai aussi pour votre philosophie ? Dans quelle crise de la philosophie traditionnelle et naturaliste s'inscrit donc votre pensée?

Ma philosophie ne s'est inscrite dans aucun contexte de dépression philosophique particulier qui permettrait à une philosophie artificialiste de se développer. II n'y a rien à chercher de ce côté-là pour la raison toute simple que je ne suis pas un philosophe artificialiste. II se trouve que j'ai été amené à aborder ces thèmes par le biais de l'étude du concept de hasard, de l'épicurisme, qui ne sont pas mes thèmes de prédilection. Les thématiques que je privilégie sont celles qui ont un rapport avec le tragique, avec les raisons de vivre ou de ne pas vivre et avec l'expérience de la joie, qui est mon souci unique et constant. Par conséquent, l'artificialisme est venu s'agréger à un train de réflexions annexes. Je cherchais à l'époque à définir une pensée qui ne se fasse pas d'illusions sur le réel et je suis tombé sur la pensée du hasard, celle du non-sens absolu qu'est le hasard, et cela m'a amené à faire un peu d'histoire de la philosophie et à constater qu'il y avait eu de grands courants artificialistes dans lesquels je me

3. L'anti-nature. Éléments pour une philosophie tragique, p. 127. 
reconnaissais pour ce chapitre-là de la philosophie mais pas pour les points qui m'intéressaient le plus directement. II n'y avait donc pas de raison particulière pour que surgisse une pensée artificialiste à ce moment précis.

C'est pourquoi vous n'employez plus par la suite le terme artificialiste?

En effet, c'est désormais pour moi du passé même si je ne reviendrais pas pour autant sur ce que j'ai dit dans L'anti-nature.

Votre ouvrage de prime jeunesse, La philosophie tragique, énonce la condition tragique dans laquelle, face à la mort, chaque être humain se trouve. De manière métaphorique, vous avez associé cette révélation tragique - à laquelle tout homme est confronté - à la surprise d'un enfant à qui, pour la première fois, on a refusé un jouet. "Si sa stupéfaction cesse, écriviezvous, [l'homme] n'est plus tragique»4. Cette stupéfaction éprouvée voilà bientôt quarante ans vous habite-t-elle toujours aujourd'hui ? Ettes-vous toujours un homme tragique?

Tout à fait, même si je ne me souviens plus de cet exemple, et pour cause. Cet ouvrage de jeunesse est tellement mal écrit que je n'ai jamais eu le courage de le relire. J'ai fait la folie de le rééditer et d'y rédiger une introduction qui me permette de dédouaner le tout jeune homme qui l'a écrit, et qui avait à l'époque dix-neuf ans quand il en a rédigé la première moitié. Je me souviens un peu plus de l'exemple d'un certain maçon mais je reste en accord avec ce sentiment. Je reconnais ce sentiment très fort qui se révèle sous une forme noire chez Cioran et sous une forme plus profonde encore chez Lucrèce qui éprouve une certaine pitié à constater que l'homme n'est pas capable d'affronter la pensée de la mort. La comparaison avec l'enfant me paraît justifiée puisqu'il y a dans la détresse enfantine devant un petit chagrin ou dans celle d'un jeune adulte devant un premier grand chagrin d'amour quelque chose

\section{La philosophie tragique, p. 20.}


qui serait comme une sorte de préparation au grand désarroi qui est de nous trouver possesseurs d'un savoir que nous ne pouvons pas assumer. Tout philosophe, si illustre soit-il, qui ne s'accorde pas sur ce point avec Cioran, Lucrèce, Nietzsche, Schopenhauer, etc. et qui ne passe par le credo absolu et sans nuance qui veut que l'homme ne soit pas capable d'accepter de penser ce qu'il sait est pour moi d'avance disqualifié. Je peux le lire, bien sûr, parce qu'il a écrit des choses géniales sur le rapport entre la forme et la matière, sur ceci ou cela, mais cela reste pour moi de l'ordre du détail. II n'en reste pas moins que sur le point que je considère comme fondamental ce philosophe a encore tout à apprendre et qu'il est loin de se douter de la profondeur de l'abîme qu'il côtoie sans le voir.

Dans ce même ouvrage de jeunesse, vous évoquiez le rôle capital à vos yeux de la joie comme seule réelle échappatoire possible au tragique de l'existence. Cette allégresse, exposée de nouveau dans nombre de vos ouvrages suivants, a-t-elle toujours un écho aussi fort dans votre vie ? Pensez-vous toujours qu'elle est l'unique remède possible au tragique?

Oui et non. Je n'ai pas changé de point de vue même si je ne suis pas persuadé que seuls les imbéciles ne changent jamais d'idée comme le disent nos hommes politiques. Le tout dernier livre que j'ai publié (Le démon de la tautologie) était une manière de clore le débat en reprenant certains thèmes de $L a$ philosophie tragique, et notamment ceux de l'exaltation de la joie de vivre et de la condamnation de la morale repérée comme machine de guerre contre le réel et la faculté de penser le réel, faculté qui ne peut être que jubilatoire. Mais ce sont vos expressions que je n'emploierais pas. La joie n'est pas du tout un remède ou une échappatoire puisque je considère à l'inverse que ce qui donne sa valeur à la joie, c'est son aptitude à ne pas éluder l'obstacle mais au contraire à intégrer la connaissance du pire à son exercice jubilatoire de la vie. C'est justement le fait que ce ne soit pas une échappatoire qui constitue la joie. Je suis ainsi d'accord avec votre idée mais non avec sa formulation. 
Dans Logique du pire, votre second ouvrage théorique, vous évoquiez la possibilité pour une "philosophie terroriste» de "faire passer le tragique de l'état inconscient à l'état conscient»5. Or, dans vos derniers textes, vous semblez croire qu'il est à jamais impossible de guérir les sectateurs d'idéologies en tout genre, enfermés à jamais dans leur dogmatisme et capables tout au plus de faire varier la source de leur idéologie mais non de s'en défaire. Est-ce à dire que votre Logique du pire ne peut que prêcher des convertis?

Je n'ai pas relu ce livre depuis longtemps mais je crois me rappeler y avoir dit explicitement qu'il est impossible de désillusionner les hommes en leur imposant un savoir qu'ils peuvent admettre sur le plan théorique ou abstrait mais qui n'imprégnera pas leur véritable sensibilité.

II me semble pourtant que c'est à partir du Réel et son double que vous dressez ce constat.

Le thème, il est vrai, a pris plus de consistance dans les ouvrages suivants. Mais il est déjà présent en filigrane dans Logique du pire où je dis clairement qu'un savoir n'est rien s'il est inutilisable et que l'homme de toute croyance, de toute idéologie, au sens courant du terme, est absolument inébranlable. J'avais déjà en tête à l'époque cette idée mais vous avez raison de dire qu'elle a pris une dimension plus importante et plus nette à partir du Réel et son double. Faire passer le tragique de l'état inconscient à l'état conscient est une impossibilité : vous en avez conscience ou bien vous n'en avez pas conscience et vous n'en aurez jamais conscience.

Quant à prêcher des convertis, je suis bien entendu d'accord et je n'ai cessé de le redire; c'est d'ailleurs pourquoi mon nombre de lecteurs est réduit. On ne peut guérir que des bien portants, c'est là pour moi une devise absolue.

5. Logique du pire. Éléments pour une philosophie tragique, p. 24. 
C'est pourquoi vous faites une différence entre les philosophesguérisseurs et les philosophes-médecins.

Exactement.

Si le réel est tautologique, comme vous le dites dans votre dernier ouvrage ${ }^{6}$, toute entreprise philosophique, en tant qu'elle est une réflexion sur la réalité, est-elle pour autant condamnée à ne rien penser ou à ne rien dire - si ce n'est une re-dite ? N'avez-vous pas peur, vous qui dénoncez sans cesse les dangers de duplication du réel, de faire de votre cuvre un double de la réalité qu'elle évoque?

Je crois que la redite est une expérience rarissime et extrêmement enrichissante à la fois, en ce qu'elle nous découvre un réel qui peut paraître banal. C'est comme si la réalité nous était redonnée et c'est pourquoi la véritable redite, que je distingue des fausses redites dans Le démon de la tautologie, me paraît être un exploit philosophique.

Cette seule redite permet d'échapper au double?

Exactement, c'est même le contraire du double. II y a le monde de l'illusion, le monde du double, et le monde du sentiment de la réalité qui est celui de la redite. II faudrait préciser cela et vous me donnez là la matière d'un nouveau livre.

Je voudrais ajouter aussi, et c'est là une remarque très générale, que ce qui me pousse à faire œuvre de philosophe n'est pas seulement cette idée que la connaissance du pire ou la plus grande lucidité vis-à-vis de soi-même me paraît être une chose "bonne" (même si ce mot me semble suspect à cause de l'usage moralisant qui en est fait parfois), mais qu'un autre ressort me fait prendre la plume, qui n'a rien à voir avec le premier, et qui est un ressort ludique. II s'agit bien évidemment du plaisir d'écrire, de ce plaisir de fabriquer et d'arranger les mots entre eux...

6. Le démon de la tautologie suivi de cinq petites pièces morales. 
Plaisir que vous évoquez dans Le choix des mots, n'est-ce pas?

En effet. Je retire deux bénéfices de cet exercice, et j'espère que mes lecteurs en ressentent un des deux. D'abord un bénéfice de salubrité publique et intellectuelle et ensuite un plaisir de fabriquer que tout artiste ressent et que tout travail bien fait nécessite. Cela me procure, dans la lutte contre les mots et la matière, une véritable jouissance, équivalente à celle du vigneron de Jules Renard qui fait de son vin un miracle de l'art. Bien entendu, on n'écrirait jamais rien si on n'avait l'idée qu'il n'y a qu'une chance sur un million que ceci serve à quelque chose. Je ne veux pas pour autant faire preuve de fausse modestie car il n'y a rien de plus agaçant à mes yeux que d'entendre un individu que la publicité a porté aux nues ne cesser de dire qu'il n'est rien du tout ou le dernier des derniers. Je ne voudrais pas donner dans ce travers.

Cette volonté d'écrire entre littérature et philosophie - André Comte-Sponville parle, lui, d'un indispensable "entre-deux" à ce propos - est-elle ce qui vous pousse à émailler vos textes de références autres que philosophiques?

Il est vrai que je n'hésite pas à recourir à des références musicales, plastiques ou littéraires (romans, théâtre, bandes dessinées, etc.). Mon atelier comprend tout. J'aime musarder et mettre ensemble des citations ou des faits d'art qu'on n'aurait pas tendance à rapprocher et à éclairer d'une même luminosité. J'aime mettre en valeur deux éléments qui paraissent en apparence lointains et qui, en fait, consonnent quand on sait les faire consonner. C'est un plaisir et en même temps une manière de faire de la philosophie qui donne une profondeur à des notions en montrant comment elles peuvent scintiller en convergence avec d'autres qui leur paraissent très opposées. Le fait d'essayer de produire une langue riche et travaillée même si mon but n'est pas de faire du Céline, du Zola, du Dostoïevski ou du Shakespeare (qui est à mes yeux plus grand de tous) - participe du plaisir d'écrire. 
D'ailleurs, vous ne faites pas de différence réelle et essentielle entre philosophie et littérature puisque vous dites également que la différence se situe principalement au niveau de l'objet et non de la méthode.

Effectivement, dans la mesure où tout est affaire de mots. Et c'est pourquoi j'aime faire un montage d'éléments provenant de casiers hétéroclites. Une critique qui pourrait m'être faite et qui ne me causerait aucun déplaisir, puisqu'elle montrerait que la personne a compris, sinon le message, du moins ma manière de procéder, serait de dire que mes livres ne sont que des citations mises bout à bout et agrémentées d'un propos personnel. Mes livres sont effectivement courts et la plupart du temps ce n'est pas moi qui parle... J'aime beaucoup imiter Luciano Berio qui fait en musique des collages de partitions différentes. Mais n'y voyez pas là une provocation! Certains pensent qu'entre deux citations de Heidegger faire intervenir un mot d'un album de Tintin et Milou est une manière de se moquer du monde, et de Heidegger en particulier. Au contraire, je ne vois pas en quoi ce serait amoindrir Heidegger que de le faire succéder ou précéder par Hergé. Je pense en outre qu'il y a une profonde pertinence dans ce rapprochement qui me sert à éclairer ce que je veux dire.

J'aimerais revenir à la notion de réel et évoquer celle de double, si prégnante dans votre œuvre. Que pensez-vous de cette volonté qu'ont certains de nos contemporains de vouloir doubler le singulier par le clonage ? Y êtes-vous farouchement opposé ? Et si oui, au nom de quoi?

Je ne suis absolument pas opposé au clonage. Le clonage peut me poser un problème purement intellectuel puisqu'il m'amène à me demander si je ne devrais pas réviser certaines expressions dont j'use à propos du réel que je définis fondamentalement comme réalité non duplicable. Le réel, avant d'être $A=A$, c'est ce qui n'a pas de double. Cela dit, je suis absolument ahuri par l'unanimité avec laquelle tous les chefs d'États et toutes les assemblées du monde, avant même d'examiner le fond du problème, ont refusé d'en traiter sérieusement. C'est 
une réaction typiquement médiévale face au diabolus in clonago. Mais quand on regarde les choses de près et qu'on reconnaît les services immenses que le clonage peut produire dans des domaines infiniment variés, l'interrogation demeure. Je n'ai pas vraiment d'opinion et, en définitive, je veux bien reconnaître que le clonage apparaisse peut-être comme une chose plus inquiétante que rassurante. Mais ce qui est absolument insensé c'est d'avoir une opinion toute faite avant même d'avoir la moindre information sur le sujet. C'est pour moi un exemple de pensée médiévale (j'entends par là une pensée rétrograde car je sais que la grande pensée médiévale est autre) et la preuve évidente que nos contemporains, avant de se demander si une chose est utile ou inutile ou encore vraie ou fausse, pensent en termes de bien et de mal et remplacent toute analyse par une question morale du type : est-ce ou non conforme à la morale? Cela m'apparaît être un comportement effarant et si je devais porter à mon tour un jugement moral, je dirais que la catégorie des gens immoraux se limite à la catégorie des moralistes.

Puisque vous évoquez la morale, parlons-en. Vous semblez proche du Marcel Conche qui voit dans le réel qui se donne à nous une somme d'événements, l'Événement par excellence, qu'il refuse lui aussi d'unifier dans un "être" ou une "nature des choses». Pourtant, vous vous en éloignez en ce qui concerne les implications pratiques de votre philosophie. Comment expliquez-vous, et ce sont là les mots de Marcel Conche lui-même qui m'a demandé de vous transmettre cette question, que quelqu'un d'aussi sérieux que l'auteur de Vivre et philosopher se trompe si radicalement sur les questions morales ? Pensez-vous que sa volonté de donner un fondement à la morale relève d'une illusion ?

Je connais très mal Marcel Conche dans le privé, encore que j'aie beaucoup conversé épistolairement avec l'homme. Disons que j'ai une vague idée de la manière dont les choses ont pu s'agencer dans son esprit et je considère comme étonnant qu'un homme aussi éminent veuille fonder la morale; c'est pour moi une contradiction dans les termes. C'est là le petit grain du génie, si vous voulez, un peu comme chez Molière, ce 
personnage du Médecin malgré lui, à qui on fait croire au début de la pièce qu'il est le plus grand médecin de la terre, ce que sa folie l'empêche de reconnaître. Je vois néanmoins avec plaisir, pour répondre par votre intermédiaire à l'expéditeur de la question, qu'il est lui-même sensible au problème que je me pose.

Dans votre Logique du pire, vous exposez nettement votre position pratique en parlant de l'indispensable tolérance du philosophe tragique, qui ne va pas pourtant jusqu'à tout tolérer puisque ce dernier agit "en arrachant, par exemple, lorsque la possibilité s'en présente, un être à la souffrance ou à la mort»?. Mais au nom de quoi agit-il ainsi s'il refuse toute conscience morale?

En tout cas, pas au nom de la conscience morale. Le fait, quand la chose est possible, d'arracher quelqu'un à la souffrance sous toutes ses formes, n'entraîne pas pour autant un quelconque moralisme. Nous avons connu des gens qui affirmaient vouloir le bien d'autrui et qui étaient très favorables à la souffrance qu'ils infligeaient, ce qui ne les a pas empêchés d'avoir le label de ceci ou de cela, et de sauveur de la terre en particulier. Face à cela, je pense à un mot de Nietzsche qui résume ma position. "Qu'est-il de plus humain ?" est-il demandé dans l'avant-dernier aphorisme du troisième livre du Gai Savoir, «Épargner la honte à quelqu'un» y est-il répondu... J'ai beaucoup de peine à répondre à votre question. En tout cas, ce n'est pas agir au nom de la morale ni au nom d'un principe. C'est de la compassion. La souffrance d'autrui m'est intolérable, n'en déplaise à mes détracteurs.

Et vous n'éprouvez pas le besoin de faire reposer cet intolérable sur un principe si je vous comprends bien...

Bien entendu et je renchérirais en disant que celui qui a besoin d'un principe pour éprouver de la compassion est un homme sur lequel je ne miserais aucune confiance. On a moins à

7. Logique du pire. Éléments pour une philosophie tragique, p. 155. 
craindre d'une franche crapule que de quelqu'un qui prétend faire le bien par devoir.

Ce qui vous sépare de Marcel Conche, mais aussi d'André Comte-Sponville qui le suit sur ce point, n'est-ce pas le fait qu'ils ont tendance à substantialiser l'homme (ils parlent de "droits de l'homme») là où vous lui refusez toute consistance ontologique?

II y a de ça même si je ne suis pas persuadé que l'homme n'existe pas, intuition qui n'est pour moi qu'une pensée hygiénique. Mais je crois que la raison qui les amène à penser ainsi est la même que celle qui a fait penser d'une manière identique beaucoup d'autres auteurs. Plus profondément, il me semble que ce qui leur répugne dans l'idée de ne pas avoir de principes moraux d'action tient à la frustration d'un besoin de certitude. II y a des gens qui pensent, en gros, qu'il suffit qu'ils s'en remettent à la vie qui passe et qu'ils sauront toujours quoi faire. Ce n'est pas un orgueil fou, c'est tout simplement de l'empirisme. II y en a d'autres, plus craintifs, qui se disent : «si je n'ai pas fait un nœud à mon mouchoir dans ma poche pour me dire "il faut être gentil", je suis perdu". II leur faut cette certitude et s'ils ne l'éprouvent pas de manière aiguë dans le domaine des pensées spéculatives, du moins l'éprouvent-ils en matière morale. Et le cas exemplaire à ce sujet c'est bien sûr Kant. Kant nous dit qu'il a renoncé à toute certitude. En réalité, il y a renoncé pour mieux asseoir une seule certitude, la certitude morale. J'associe cela à l'idée de l'homme qui aborde l'existence content parce qu'il a fait un nœud à son mouchoir. Moi, je n'ai pas fait de nœud à mon mouchoir et je pense que cela vaut mieux.

II me semble que Conche valorise en effet ce côté kantien, mais Comte-Sponville me paraît s'en être détaché. En effet, il ne fait pas reposer la morale sur des principes mais sur des exemples, et en cela il me paraît être spinoziste puisqu'il peut dire, avec Spinoza, la chose suivante : “j'entendrai donc par 
bon [...] ce que nous savons avec certitude qui est un moyen de nous rapprocher de plus en plus du modèle de la nature humaine que nous nous proposons. Par mauvais, au contraire, ce que nous savons avec certitude qui nous empêche de reproduire ce modèle. Nous dirons, en outre, les hommes plus ou moins parfaits, suivant qu'ils se rapprocheront plus ou moins de ce même modèle»8. Qu'en pensez-vous ?

Il est vrai qu'il joue plutôt sur le côté sage et non théorique de la chose. II me semble en général que Comte-Sponville est plus analyste et Conche plus historien. Mais je ne suis pas là pour parler de personnes que tout le monde connaît de nos jours. En effet, par le passé, il n'en allait pas de même. Conche a été très persécuté par l'Université et il n'a été reconnu comme philosophe qu'après sa retraite, ce qui est tout à fait scandaleux. Bien qu'il soit également un excellent analyste, il représente d'abord et avant tout un apport très important en histoire de la philosophie et notamment, même si je ne suis pas toujours d'accord sur ce point avec ses thèses, dans l'évocation qu'il fait des grands philosophes présocratiques et hellénistiques. C'est un homme extrêmement instruit, qui a appris le grec très tard et un connaisseur extrêmement pointu de tout ce qui concerne des domaines représentés par des philosophes mineurs. Son livre sur Pyrrhon en est un bon exemple, même s'il paraît que les sceptiques purs contestent cette version du pyrrhonisme. C'est en tout cas un livre passionnant, qui renouvelle le débat, ce que Conche a fait souvent. J'ai d'ailleurs acheté le Dictionnaire des philosophes parce qu'il y a inclus des notices très drôles sur des auteurs mineurs qu'il est le seul à connaître.

Mais pour en revenir à votre question je dirais que, sur le plan éthique, ce sont tous deux des kantiens. André ComteSponville, bien qu'il veuille être spinoziste, est avant tout bel et bien kantien. Je ne sais pas si c'est ce que vous cherchiez, mais vous avez réussi en tout cas à me le faire dire. Ceci dit, ce

8. Spinoza, Éthique, in CEuvres, éd. Appunh, t. III, Paris, GF-Flammarion, 1961, p. 219. 
n'est pas une injure. C'est une des nombreuses façons possibles de donner une réponse aux grands problèmes de la vie et c'est cette réponse kantienne qu'ils ont fondamentalement adoptée.

Il me semble néanmoins qu'André Comte-Sponville, dans son article très drôle sur la morale d'Arsène Lupin', morale qu'il oppose à celle de Kant, est loin d'être kantien...

Si ce nouveau Comte-Sponville m'a beaucoup amusé, je reste persuadé que sur le fond, malgré les réflexions distanciées qui sont faites à propos de la morale et qui sont très justes, ce gentleman-cambrioleur pourrait bien vite être classé dans la catégorie des kantiens. Comte-Sponville cite d'ailleurs la réponse d'Arsène Lupin à la question suivante : "Alors vous faites le bien aussi? - Quand j'ai le temps. Et puis ça m'amuse»10. Cet article est drôle et amusant mais ce n'est pas un manquement au kantisme. Cette petite récréation montre simplement que l'on ne peut pas faire du Kant toute la journée.

Dans Le démon de la tautologie, vous fustigez, et avec raison, certains excès du moralisme écologique. Ne trouvez-vous pas quand même malheureux le gâchis ontologique dont l'homme moderne se rend coupable et qui tend peu à peu à faire triompher l'inanimé sur le vivant (pensée que l'on trouve par exemple chez Marcel Conche)? Pensez-vous là encore que le philosophe tragique ne doit pas se prononcer et encore moins militer?

Je ne pense pas que le philosophe tragique puisse militer pour quoi que ce soit. Cela dit, en ce qui concerne l'écologie, il est de fait que très souvent elle est profondément morale et repose sur des raisons aussi absurdes que celles qui ont fait rejeter le clonage sans examen d'aucune sorte. Il est certain aussi qu'elle est fortement, à mon avis, engagée dans la psychopathologie

9. "La morale d'Arsène Lupin ou Arsène Lupin contre Kant", dans Arsène Lupin gentilhomme-philosopheur, Paris, Le Félin, 1996, p. 45-66.

10. Ibid., p. 45. 
parce qu'elle est très souvent compensatoire. C'est souvent l'équivalent d'une secte, d'une certitude qu'on a pas trouvée, d'un manque, d'un mari ou d'une femme qu'on a pas rencontrés, d'un amour resté lettre morte, et ce caractère compensatoire me paraît évident. II suffit de rentrer dans un restaurant écologique pour s'en rendre compte. Dans ce lieu où on est censé vivre en dehors de tous les poisons de la vie moderne, voyez ces gens au bord de la mort, épuisés et exsangues et d'une humeur exécrable. L'écologie me semble donc engagée au mieux dans de la morale et dans ce que la morale a d'aveugle, au pire dans une pathologie. Un psychanalyste vous en dirait plus long là-dessus...

J'ouvre une parenthèse pour vous raconter une histoire amusante. J'ai suivi une émission à la télévision où un de nos amuseurs nationaux s'opposait à un grand restaurant du Var que je connais bien au nom de l'écologie et du végétarisme. II y avait une dame sur le plateau, mère de cinq enfants, et qui, pour n'avoir mangé que de la salade, était pourtant bien en chair. Cette dame nous apprend que ses enfants adorent naturellement les légumes et que jamais ils ne se retourneraient devant l'étalage d'un boucher (elle semblait croire que ce qui relève de l'acquis est au fond naturel). Le présentateur lui demande alors si un tel fait ne relève pas de l'habitude, de l'ethos, vieux problème philosophique s'il en est. Cette dame a eu une réponse splendide, fabuleuse, digne de certaines répliques de la princesse de Monaco (celle qui disait que les animaux étaient des hommes comme les autres) : "Ah non, pas les miens, mes cinq enfants sont végétariens de naissance».

Plaisanterie mise à part, ce phénomène qui contribue à faire triompher l'inanimé sur le vivant est bien sûr inquiétant. Mais je ne vois pourtant pas en quoi le philosophe tragique aurait à s'en mêler. Je serais de plus plutôt optimiste à propos de ce problème qui peut, contrairement à d'autres problèmes, trouver une solution. Ce qui est encore plus remarquable c'est ce que du mal viendra le bien puisque c'est, me semble-t-il, des moyens mis en œuvre par la technique qui a pollué que viendront les moyens les plus sûrs et les plus fiables de 
progressivement dépolluer. Donc, je crois qu'il ne faut pas s'affoler et faire confiance à la science tout en étant vigilant. N'importe qui pourrait en dire de même d'ailleurs.

Pour vous, l'écologie contemporaine c'est du Pline l'Ancien remis au goût du jour...

En effet, si vous lisez Pline, et j'ai été moi-même stupéfait lors de cette lecture, le monde n'a plus que quinze jours à vivre ! Cela doit nous donner un peu de sérénité...

Une de mes questions précédentes employait le verbe militer. J'aimerais savoir quels sont les rapports que vous entretenez avec la politique, si du moins la politique vous intéresse. La considérez-vous comme réellement inutile (et dans ce cas le philosophe devrait se refuser à en faire) ou bien lui attribuezvous une quelconque valeur?

Je crois que la réponse la plus sincère serait de vous dire que c'est un domaine qui ne m'intéresse absolument pas. La théorie du pouvoir, la philosophie politique, comme on la trouve chez Hobbes, chez Machiavel ou chez Spinoza, m'intéresse beaucoup. J'ai d'ailleurs consacré une cinquantaine de pages du Philosophe et les sortilèges au pouvoir. Je ne m'y désintéresse donc pas tout à fait. Mais je ne m'y connais pas vraiment et ne peux ici qu'improviser car ce n'est pas ma spécialité. J'ai toutefois l'impression que les mécanismes du pouvoir se sont profondément modifiés depuis la fin de la Seconde Guerre Mondiale. II y a aujourd'hui un phénomène d'internationalisation qui fait que, finalement, j'ai le sentiment que le pouvoir se développe de plus en plus malheureusement dans le privé en quittant peu à peu la sphère publique. Ce que je veux dire c'est que les pouvoirs publics ont de moins en moins de pouvoir. Un Président de la République actuel, dans notre Cinquième République, surtout en période de cohabitation, a moins de pouvoir qu'un Président de la Quatrième République, qui n'avait pourtant qu'un pouvoir très restreint. Et ceci se renforce avec la perte de souveraineté progressive due à la constitution d'une 
souveraineté européenne. Mais il y a une interdépendance des moyens techniques qui est telle que le pouvoir arbitraire, celui des Xerxès par exemple, a tendance à devenir de moins en moins possible sauf envers des populations moins contrôlées que les nôtres (prenez par exemple les épouvantables massacres en Algérie ou au Rwanda). En revanche, le père ou la mère de famille et tous ceux qui sont responsables d'une certaine tyrannie domestique (et non politique), ne sont pas contrôlés, à moins que certaines affaires familiales n'éclatent au grand jour (les scandales reliés à la pédophilie aujourd'hui). Je crois que si je devais militer contre le pouvoir ce serait contre le pouvoir familial, mais non contre la famille, qui a pour moi plus de bon que de mal. Je ne suis pas du tout un anarchiste.

Mais si je devais avoir un engagement politique prioritaire aujourd'hui, ce serait celui m'amenant à militer en faveur de l'indépendance du Québec, et ce pour le Québec bien sûr, mais aussi pour des raisons qui, je l'avoue, sont intéressées. Je pense qu'une souveraineté indépendante aurait plus de chances de préserver la langue française encore présente en Amérique du Nord. Je ne suis donc pas tout à fait dénué d'un certain nationalisme.

J'aimerais pour finir cet entretien évoquer une citation du Réel et son double que je trouve particulièrement belle. Vous écri-viez : "Sois ami du présent qui passe : le futur et le passé te seront donnés par surcroît»11. Êtes-vous toujours de ce sentiment?

Oui, bien sûr, et si je devais garder une phrase de tout ce que j'ai écrit, je garderais celle-là.

Sébastien Charles

Département de philosophie

Université d'Ottawa

11. Le réel et son double. Essai sur l'illusion, p. 82. 


\section{BIBLIOGRAPHIE DE CLÉMENT ROSSET}

La philosophie tragique, Paris, P.U.F., 1960.

Lettre sur les chimpanzés, Paris, NRF-Gallimard, 1965.

Schopenhauer, philosophe de l'absurde, Paris, P.U.F., "Quadrige», 1967.

L'esthétique de Schopenhauer, Paris, P.U.F., «Quadrige», 1969.

Logique du pire. Éléments pour une philosophie tragique, Paris, P.U.F., "Bibliothèque de philosophie contemporaine», 1971.

L'anti-nature. Éléments pour une philosophie tragique, P.U.F., «Philosophie d'aujourd'hui», 1973.

Le réel et son double. Essai sur lillusion, Paris, NRF-Gallimard, 1976.

Le réel. Traité de l'idiotie, Paris, Éditions de Minuit, “Critique», 1977.

L'objet singulier, Paris, Éditions de Minuit, “Critique», 1979.

La force majeure, Paris, Éditions de Minuit, "Critique», 1983.

Le philosophe et les sortilèges, Paris, Éditions de Minuit, "Critique», 1985.

Le principe de cruauté, Paris, Éditions de Minuit, "Critique», 1988.

Principes de sagesse et de folie, Paris, Éditions de Minuit, "Critique», 1991.

En ce temps-là. Notes sur Althusser, Paris, Éditions de Minuit, 1992.

Joyful Cruelty. Towards a Philosophy of the Real, Oxford University Press, 1993 (translated and edited by David F. Bell).

Le choix des mots suivi de La joie et son paradoxe, Paris, Éditions de Minuit, 1995.

Le démon de la tautologie suivi de Cinq petites pièces morales, Paris, Éditions de Minuit, «Paradoxe», 1997. 